\title{
Editorial zum Themenschwerpunkt Femoroazetabuläres Impingement
}

In den letzten Jahren ist das femoroazetabuläre Impingement zunehmend in den Fokus der Hüftchirurgen gerückt. Nach den maßgeblichen Arbeiten von Professor Reinhold Ganz über die Pathomorphologie und Aufzeigung von Behandlungsmöglichkeiten sind verschiedene Therapieansätze entwickelt worden. Neben der als Goldstandard anzusehenden offenen Operationstechnik mit Trochanterosteotomie und Hüftluxation in der von Ganz et al. entwickelten Technik sind arthroskopische Verfahren, kombiniert arthroskopische und offene minimalinvasive sowie rein offene minimalinvasive Techniken entwickelt worden.

In diesem Schwerpunktheft werden die traditionell offene Technik mit Trochanterosteotomie, die arthroskopische Technik und die Technik über einen minimalinvasiven anterioren Zugang dargestellt. Die verschiedenen Vor- und Nachteile dieser Verfahren werden besprochen sowie Ergebnisse aus Zentren präsentiert.
Die genannten Techniken sind nicht als allein miteinander konkurrierende Therapieverfahren zur Behandlung des femoroazetabulären Impingements zu sehen, sondern dürften aufgrund ihrer Vorteile Indikationen bei unterschiedlichen Befundkonstellationen haben. Die Aufgabe zukünftiger Arbeiten wird es sein, neben der ständigen Weiterentwicklung der Techniken ein differentialindikatorisches Vorgehen herauszuarbeiten, wann welche Operationstechnik zu bevorzugen ist bzw. gegenüber den anderen beiden ihre Vorteile hat. Allen drei Operationstechniken bleibt jedoch gemein, dass sie chirurgisch anspruchsvoll sind und eine nicht zu unterschätzende Lernkurve bedingen. Es ist daher zu fordern, dass die Behandlung des femoroazetabulären Impingements Zentren vorbehalten bleibt, die neben allen diagnostischen Methoden (wie Arthro-MRT) über die entsprechenden operativen Erfahrungen verfügen.

Bernd Fink 